\title{
过氧化氢：小分子，大作用
}

李厚金 ${ }^{*}$, 李金珊, 陈六平

中山大学化学学院, 广州 510275

摘要: 过氧化氢, 结构微妙, 用途广泛, 在漂白、医药、化工、环保、食品工业、火箭燃料等多行业发挥重要作用。本 文在简述过氧化氢的结构、性质和合成的基础上, 重点讨论其生物学功能、检测方法、应用、安全性等, 增加人们对过 氧化氢的进一步了解。

关键词: 过氧化氢; 化学合成; 生物学功能; 检测方法; 应用; 安全性

中图分类号: G64; O6-1

\section{Hydrogen Peroxide: Small Molecule with Big Functions}

\author{
Houjin Li ${ }^{*}$, Jinshan Li, Liuping Chen \\ School of Chemistry, Sun Yat-Sen University, Guangzhou 510275, China.
}

Abstract: Hydrogen peroxide is a small molecule with a fine structure and wide applications. It plays important roles in bleaching, medicine, the chemical industry, environmental protection, food industry, rocket fuel etc. Here, in addition to providing a brief comprehensive introduction on the structure, chemical properties and syntheses of hydrogen peroxide, we primarily present its biological functions, detection methods, applications and safety, with the aim of enhancing the understanding of this smart molecule.

Key Words: Hydrogen peroxide; Chemical synthesis; Biological function; Detection method; Application; Safety

氢氧化合物有水、过氧化氢( hydrogen peroxide, $\mathrm{H}_{2} \mathrm{O}_{2}$ ) 和超氧化氢( hydroperoxyl, $\mathrm{HO}_{2} \cdot$ )。水的重 要性冊庸多言。超氧化氢是自由基, 性质活泼 ${ }^{[1]}$ 。 $\mathrm{H}_{2} \mathrm{O}_{2}$ 介于二者之间, 自 19 世纪初被发现后, 已在 漂白、化工、食品、环保、医疗卫生、军工等领域广泛应用。2007 年, 水和 $\mathrm{H}_{2} \mathrm{O}_{2}$ 均入选 “ 100 种最 重要的化学物质” 之列 ${ }^{[2]}$ 。本文在简要介绍 $\mathrm{H}_{2} \mathrm{O}_{2}$ 的结构、化学性质与合成的基础上, 重点讨论其生 物学功能、检测方法、应用、安全性等。

\section{$1 \mathrm{H}_{2} \mathrm{O}_{2}$ 的存在与发现}

据推测, 约 40 亿年前地球上就已经出现 $\mathrm{H}_{2} \mathrm{O}_{2}$, 那时没有臭氧层, 在高强度紫外线照射下水发 生光化学反应产生 $\mathrm{H}_{2} \mathrm{O}_{2}{ }^{[3]}$ 。在元古代, 地球经历过几乎完全被冰雪覆盖(雪球地球)的深度冰冻状态, 弱水文循环和水的光化学反应导致 $\mathrm{H}_{2} \mathrm{O}_{2}$ 持续产生并储存在冰中, 冰融化时 $\mathrm{H}_{2} \mathrm{O}_{2}$ 被释放到海洋和大 气中, 并引起全球氧化事件 ${ }^{[4]}$ 。早期的生命就开始过氧化氢酶和超氧化物歧化酶的进化设计, 以促

收稿: 2020-06-08; 录用: 2020-07-24; 网络发表: 2020-08-26

“通讯作者, Email: ceslhj@mail.sysu.edu.cn

基金资助: 广东省科技计划项目(2019B070702003); 广东省高等教育教学改革项目; 中山大学本科教学改革研究项目 
使 $\mathrm{H}_{2} \mathrm{O}_{2}$ 分解。自然界中 $\mathrm{H}_{2} \mathrm{O}_{2}$ 无处不在, 在雨、露、云、雪、空气等所有地表水中 ${ }^{[5]}$, 甚至在寒冷、 干燥、低氧的火星和伽利略欧罗巴卫星地表气体成分中都有 $\mathrm{H}_{2} \mathrm{O}_{2}{ }^{[4]}$ 。1818 年, 法国化学家 LouisJacques Thenard 研究碱土金属时, 将钡燃烧的产物过氧化钡 $\left(\mathrm{BaO}_{2}\right)$ 溶解在水中, 得到了 $\mathrm{H}_{2} \mathrm{O}_{2}$, 这是 科学家首次发现 $\mathrm{H}_{2} \mathrm{O}_{2}{ }^{[6]}$ 。

\section{$2 \mathrm{H}_{2} \mathrm{O}_{2}$ 的结构}

为解决 $\mathrm{H}_{2} \mathrm{O}_{2}$ 分子的结构问题, 曾经历了反复推测、理论计算、实验验证的工作, 不同方法得出 的结果也存在差异。目前公认的 $\mathrm{H}_{2} \mathrm{O}_{2}$ 的结构数据是用电子衍射技术分析其气体和 X-射线单晶衍射 技术分析其晶体得出的。 $\mathrm{H}_{2} \mathrm{O}_{2}$ 的分子结构非常奇妙, 它不是平面形而是立体的。晶体中, $\mathrm{O}-\mathrm{O}$ 键 和 $\mathrm{O}-\mathrm{H}$ 键键长分别为 $145.3 \mathrm{pm}$ 和 $98.8 \mathrm{pm}, \mathrm{O}-\mathrm{O}$ 键和 $\mathrm{O}-\mathrm{H}$ 键间夹角为 $102.7^{\circ}, 2$ 个 $\mathrm{O}-\mathrm{H}$ 键所 在平面的夹角 (二面角)为 $90.2^{\circ}$ (图 1) ${ }^{[7]}$ 。随状态和所处的环境不同, $\mathrm{H}_{2} \mathrm{O}_{2}$ 结构中二面角发生显著变 化。 $\mathrm{H}_{2} \mathrm{O}_{2}$ 的分子间由氢键缔结, 缔合程度和极性都比 $\mathrm{H}_{2} \mathrm{O}$ 大, $\mathrm{H}_{2} \mathrm{O}_{2}$ 和 $\mathrm{H}_{2} \mathrm{O}$ 的偶极矩分别为 2.16 和 $1.87^{[8]}$ 。由于 $\mathrm{H}_{2} \mathrm{O}_{2}$ 分子结构的非对称性及过氧键的存在, 造就了 $\mathrm{H}_{2} \mathrm{O}_{2}$ 分子独特的性质和用途。

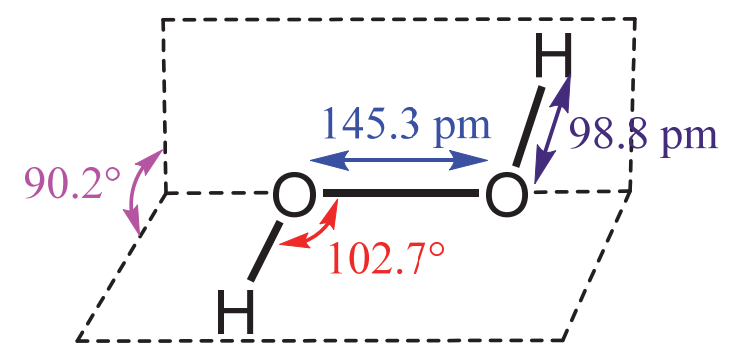

图 1 固态 $\mathrm{H}_{2} \mathrm{O}_{2}$ 分子的立体结构

\section{$3 \mathrm{H}_{2} \mathrm{O}_{2}$ 的化学性质}

$\mathrm{H}_{2} \mathrm{O}_{2}$ 的性质主要从酸碱性、分解性和氧化还原性三方面讨论。

在水溶液中, $\mathrm{H}_{2} \mathrm{O}_{2}$ 的 2 个氢原子均可解离成氢离子, 但解离常数很小, 故酸性很弱, 可与碱性 物质发生反应。若储存条件得当, 无杂质污染, $\mathrm{H}_{2} \mathrm{O}_{2}$ 可长时间保存而很少分解。实际上, $\mathrm{H}_{2} \mathrm{O}_{2}$ 的性 质极不稳定, 遇热、光、粗䊁表面, 乃至微量重金属离子(如 $\mathrm{Fe}^{3+} 、 \mathrm{Fe}^{2+} 、 \mathrm{Cu}^{2+} 、 \mathrm{Mn}^{2+} 、 \mathrm{Ca}^{2+} 、 \mathrm{Mg}^{2+} 、$ $\mathrm{Cr}^{3+} 、 \mathrm{Co}^{2+}$ 等)及其他杂质, 均会迅速发生分解:

$$
\begin{aligned}
& 2 \mathrm{H}_{2} \mathrm{O}_{2}(\mathrm{l}) \rightarrow 2 \mathrm{H}_{2} \mathrm{O}(\mathrm{l})+\mathrm{O}_{2}(\mathrm{~g}) \\
& \Delta_{\mathrm{r}} H_{\mathrm{m}}^{\ominus}(298.15 \mathrm{~K})=-98.05 \mathrm{~kJ} \cdot \mathrm{mol}^{-1} ; \quad \Delta_{\mathrm{r}} G_{\mathrm{m}}^{\ominus}(298.15 \mathrm{~K})=-116.78 \mathrm{~kJ} \cdot \mathrm{mol}^{-1}
\end{aligned}
$$

$\mathrm{H}_{2} \mathrm{O}_{2}$ 分解是放热反应, 热力学倾向很大。纯 $\mathrm{H}_{2} \mathrm{O}_{2}$ 常温常压下可自发分解。 $\mathrm{H}_{2} \mathrm{O}_{2}$ 的 “稳定性” 源于动力学因素。 $\mathrm{H}_{2} \mathrm{O}_{2}$ 的氧化还原性与过氧键键能小、易断裂有关, 既是强氧化剂, 也有显著的还 原性, 所以应用很广。例如, 古代画作的处理, 作画采用的颜料铅白 $\left[2 \mathrm{PbCO}_{3} \cdot \mathrm{Pb}(\mathrm{OH})_{2}\right]$ 受侵蚀变黑 $(\mathrm{PbS})$, 利用 $\mathrm{H}_{2} \mathrm{O}_{2}$ 的氧化性, 小心处理可使 $\mathrm{PbS}$ 转化为白色的 $\mathrm{PbSO}_{4}$ 。如果遇到更强的氧化剂, 它 则表现还原性, 如酸性条件下可被高锰酸根离子 $\left(\mathrm{MnO}_{4}^{-}\right)$氧化成氧气, 这个反应可用于 $\mathrm{H}_{2} \mathrm{O}_{2}$ 的定量 分析; 它可把氯气还原成氯离子, 工业上用于氯气的清除。

在有机合成领域, $\mathrm{H}_{2} \mathrm{O}_{2}$ 是清洁氧化剂, 在温和条件下, 利用催化剂可以实现 $\mathrm{C}-\mathrm{H}$ 氧化(羟基化 和酮基化)、烯烃的高效环氧化和顺式双差基化反应、Baeyer-Villiger 氧化反应, 以及硫氧化反应 ${ }^{[9,10]}$ 。 催化剂以铝、锰、铼、铇、钛、铁、钼等金属最为常见; 非金属催化剂以酚、胺、含氟化合物为主。 在生物体内，抗氧化系统产生的过氧化氢酶(CAT)可以促使 $\mathrm{H}_{2} \mathrm{O}_{2}$ 迅速分解。

利用 $\mathrm{H}_{2} \mathrm{O}_{2}$ 及其过氧基团在一定条件下的“惰性”, 可进行某些重金属含氧离子的检测, 如 $\mathrm{Cr}_{2} \mathrm{O}_{7}^{2-}$ 、 钛酰离子 $\mathrm{TiO}^{2+} 、 \mathrm{VO}_{4}^{3-}$ 等。这些物种与 $\mathrm{H}_{2} \mathrm{O}_{2}$ 作用, 可分别形成蓝色过氧化铬 $\mathrm{CrO}_{5}$, 在酸性强的溶液 中显红色、在稀酸或中性溶液中显橙黄色的配合物 $\left[\mathrm{TiO}\left(\mathrm{H}_{2} \mathrm{O}_{2}\right)\right]^{2+}$, 在碱性至弱酸性可存在的黄色 
$\left[\mathrm{VO}_{2}\left(\mathrm{O}_{2}\right)_{2}\right]^{3-}$ 和强酸中红棕色的 $\left[\mathrm{V}\left(\mathrm{O}_{2}\right)\right]^{3+}$ 。

\section{$4 \mathrm{H}_{2} \mathrm{O}_{2}$ 的制备方法}

可通过化学、电化学、酶或光催化的方法制备 $\mathrm{H}_{2} \mathrm{O}_{2}$, 主要有葱醌法、电解-水解法、过氧化物 法、异丙醇法、氧还原法、水氧化法、氢氧直接合成法等 ${ }^{[11]}$ 。

\section{1 葱醌法}

此法已有 70 多年历史, 仍是目前大规模生产 $\mathrm{H}_{2} \mathrm{O}_{2}$ 的方法, 所得 $\mathrm{H}_{2} \mathrm{O}_{2}$ 占其总产量的 $95 \%$ 以上。 葱醌法示意图见图 2, 主要过程如下: 在有机溶剂(醇、酯或重芳烃)中, 采用 $\mathrm{Pd} / \mathrm{Al}_{2} \mathrm{O}_{3}$ 或 $\mathrm{Ni} / \mathrm{B}$ 做催 化剂, 以烷基(乙基、丁基或戊基)葱醌为工作载体, 使之加氢生成氢化葱醌; 氢化后的产物转入分离 室, 除尽氢气, 得纯净的氢化葱醌; 氢化蒽醌与空气或氧气反应, 得到 $\mathrm{H}_{2} \mathrm{O}_{2}$, 同时工作液内生成了 烷基葱醌, 经净化处理, 再送回氢化室, 循环使用。粗产品经除氧, 用水抽提、纯化, 可得高浓度 $\mathrm{H}_{2} \mathrm{O}_{2}$ 。

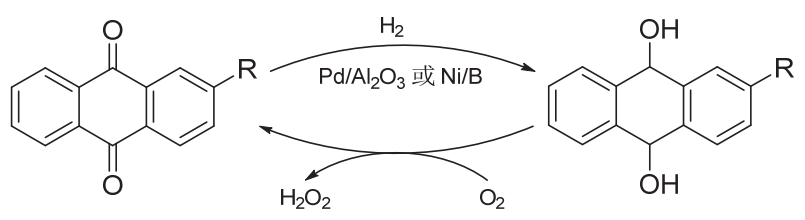

图 2 蒽醌法制备 $\mathrm{H}_{2} \mathrm{O}_{2}$

葸醌法中, 氧气和氢气不直接接触, 安全性高, 技术成熟, 能耗少, 成本低、效率高; 缺点是 过度使用有机溶剂, 且有机溶剂和 $\mathrm{H}_{2} \mathrm{O}_{2}$ 萃取液会相互污染, 均需多步提纯, 钯催化剂昂贵, 易结 块、破碎并会中毒。此外，葱醌会发生非选择性氢化反应，需周期性更换 ${ }^{[12]}$ 。

\section{2 电解-水解法}

电解法包括过硫酸法、过硫酸钾法和过硫酸铵法, 电解液可循环使用, 实际消耗水。过硫酸法 对应的电极反应和过程如下:

石墨阴极: $2 \mathrm{H}^{+}+2 \mathrm{e}^{-} \rightarrow \mathrm{H}_{2} ; \quad$ 铂阳极: $2 \mathrm{SO}_{4}^{2-}-2 \mathrm{e}^{-} \rightarrow \mathrm{S}_{2} \mathrm{O}_{8}^{2-}$

所得过二硫酸溶液在细长的管道内被外部蒸汽加热, 水解生成 $\mathrm{H}_{2} \mathrm{O}_{2}$ :

$$
\mathrm{H}_{2} \mathrm{~S}_{2} \mathrm{O}_{8}+\mathrm{H}_{2} \mathrm{O} \rightarrow \mathrm{H}_{2} \mathrm{SO}_{4}+\mathrm{H}_{2} \mathrm{SO}_{5} ; \quad \mathrm{H}_{2} \mathrm{SO}_{5}+\mathrm{H}_{2} \mathrm{O} \rightarrow \mathrm{H}_{2} \mathrm{SO}_{4}+\mathrm{H}_{2} \mathrm{O}_{2}
$$

经提纯、浓缩后得到较高浓度的 $\mathrm{H}_{2} \mathrm{O}_{2}$ 溶液。

采用如上类似的电极, 过硫酸钾法和过硫酸铵法中, 均电解硫酸氢铵饱和溶液。前者用硫酸氢 钾处理, 得过硫酸钾沉淀, 沉淀加入硫酸, 通入水蒸气, 蒸馏、冷凝后, 得到较纯的 $\mathrm{H}_{2} \mathrm{O}_{2}$ :

$$
2 \mathrm{NH}_{4} \mathrm{HSO}_{4} \rightarrow\left(\mathrm{NH}_{4}\right)_{2} \mathrm{~S}_{2} \mathrm{O}_{8}+\mathrm{H}_{2} ; \quad\left(\mathrm{NH}_{4}\right)_{2} \mathrm{~S}_{2} \mathrm{O}_{8}+2 \mathrm{KHSO}_{4} \rightarrow \mathrm{K}_{2} \mathrm{~S}_{2} \mathrm{O}_{8} \downarrow+2 \mathrm{NH}_{4} \mathrm{HSO}_{4}
$$

所得硫酸氢铵溶液经净化处理可重复使用。电流效率和水解效率较高, 20 世纪初曾用于工业生 产, 但步骤多, 投入大 ${ }^{[12]}$ 。过硫酸铵法是 20 世纪上半叶工业生产 $\mathrm{H}_{2} \mathrm{O}_{2}$ 的主要方法, 电解硫酸氢铵 饱和溶液所得过二硫酸铵在负压下水解得到 $\mathrm{H}_{2} \mathrm{O}_{2}$ 粗产品:

$$
\mathrm{S}_{2} \mathrm{O}_{8}^{2-}+\mathrm{H}_{2} \mathrm{O} \rightarrow \mathrm{HSO}_{4}^{-}+\mathrm{HSO}_{5}^{-} ; \quad \mathrm{HSO}_{5}^{-}+\mathrm{H}_{2} \mathrm{O} \rightarrow \mathrm{HSO}_{4}^{-}+\mathrm{H}_{2} \mathrm{O}_{2}
$$

经除酸、蒸馏、浓缩、精馏等提纯操作, 得到高品质的 $\mathrm{H}_{2} \mathrm{O}_{2}$ 。过硫酸铵法能耗有所降低, 是 3 种电解方法中最为经济的，但由于仍使用贵金属铂作电极，大规模生产受限，现基本被淘汰 ${ }^{[13]}$ 。

\section{3 异丙醇法}

异丙醇与氧气或空气反应生成 $\mathrm{H}_{2} \mathrm{O}_{2}$ 和丙酮。采用钒、钿、铇、铂、钯以及钉化合物作催化剂, 或再辅以光照。设备简单、操作方便、反应温和、安全性高, 但异丙醇用量大, 两种产品分离纯化 困难, 经济性欠佳 ${ }^{[14,15]}$ 。

\section{4 电化学氧还原法}

使用碳、活性物质、纤维素骨架制成气体扩散电极, 将空气中的氧气迅速而大量地溶于碱性电 
解质溶液, 通电后氧气在阴极上被还原成 $\mathrm{HO}_{2}^{-}$:

阴极: $\mathrm{O}_{2}+\mathrm{H}_{2} \mathrm{O}+2 \mathrm{e}^{-} \rightarrow \mathrm{HO}_{2}^{-}+\mathrm{OH}^{-}$;

阳极: $4 \mathrm{OH}^{-} \rightarrow \mathrm{O}_{2}+2 \mathrm{H}_{2} \mathrm{O}+4 \mathrm{e}^{-}$

$\mathrm{HO}_{2}^{-}$再用热法磷酸处理, 得 $\mathrm{H}_{2} \mathrm{O}_{2}$ 。该法电极吸附氧的能力不强, 氧气未得到有效利用, 反而因 空气气泡流入而增加了反应器的电阻。催化剂开发、隔膜选择、电极修饰以及反应器优化仍待解决 ${ }^{[16]}$ 。

4.5 光电化学水氧化法

该法是基于双电子途径的光催化水裂解, 在碳酸氢盐 $\left(\mathrm{HCO}_{3}^{-}\right)$电解液中, 水通过金属氧化物电极 在阳极被氧化生成 $\mathrm{H}_{2} \mathrm{O}_{2}: 2 \mathrm{H}_{2} \mathrm{O} \rightarrow \mathrm{H}_{2} \mathrm{O}_{2}+2 \mathrm{H}^{+}+2 \mathrm{e}^{-}$; 阴极生成 $\mathrm{H}_{2}: 2 \mathrm{H}_{2} \mathrm{O}+2 \mathrm{e}^{-} \rightarrow \mathrm{H}_{2}+2 \mathrm{OH}^{-}$。

在起始 $\mathrm{HCO}_{3}^{-}$浓度为 $2 \mathrm{~mol} \cdot \mathrm{L}^{-1}$ 时, 调节 $\mathrm{pH}=7-8,35 \mathrm{~mL}$ 水溶液中 $\mathrm{H}_{2} \mathrm{O}_{2}$ 的累积量可达 $2 \mathrm{mmol} \cdot \mathrm{L}^{-1}$ 。 反应原料为水, 驱动能量由太阳光提供。但它与氧的 4 电子反应具有竞争关系, 反应机理仍在探索 中，若能投入生产开发，经济价值可期待 ${ }^{[17,18]}$ 。

\section{6 直接合成法}

即氢气和氧气直接反应得到 $\mathrm{H}_{2} \mathrm{O}_{2}, 1914$ 年就开始研究, 因其具有原子经济性, 被认为是前景最 好的合成方法。通常的条件是低温 $\left(0-25^{\circ} \mathrm{C}\right)$ 和高压 $(1-10 \mathrm{MPa})$, 关键是催化剂的选用。可选择负载型 催化剂如 $\mathrm{Pd} 、 \mathrm{Au} 、 \mathrm{Pt} 、 \mathrm{Ni} 、 \mathrm{Zn}$ 或基于 $\mathrm{Pd}$ 的双金属催化剂等。氧气和氢气在催化膜两侧通过渗透接 触反应, 可用水、乙醇或者甲醇等做溶剂。难以工业应用的障碍在于: 安全性差, 氢氧混合物在 $4 \%-$ 94\% (物质的量浓度)浓度范围内都有爆炸性, 对工艺条件及设备要求苛刻; 反应的有效性低, 所用催 化剂能同时驱动 $\mathrm{H}_{2} \mathrm{O}_{2}$ 分解和氢氧燃烧反应, 选择性差; 低温反应, 催化剂活性低, 生产效率不高 ${ }^{[19,20]}$

此外, 还可通过燃料电池法和等离子体法来实现氢氧直接合成 $\mathrm{H}_{2} \mathrm{O}_{2}{ }^{[21]}$; 也有利用 $\mathrm{O}_{2} 、 \mathrm{CO} 、 \mathrm{H}_{2} \mathrm{O}$ 反应生成 $\mathrm{H}_{2} \mathrm{O}_{2}$ 和 $\mathrm{CO}_{2}$ 的尝试 ${ }^{[22]}$ 。

\section{5 生命体中的 $\mathrm{H}_{2} \mathrm{O}_{2}$}

\section{$5.1 \mathrm{H}_{2} \mathrm{O}_{2}$ 的产生}

几乎所有有氧生物细胞内都存在 $\mathrm{H}_{2} \mathrm{O}_{2}$, 生物体内 $\mathrm{O}_{2}$ 在有氧呼吸和光合作用等生命活动过程中, 由细胞内获取电子和质子, 在超氧化物歧化酶(SOD)作用下, 生成 $\mathrm{H}_{2} \mathrm{O}_{2}$ 。

$$
\mathrm{O}_{2} \stackrel{\mathrm{e}^{-}}{\rightarrow} \cdot \mathrm{O}_{2}^{-} \stackrel{\text { sOD, } \mathrm{e}^{-}+2 \mathrm{H}^{+}}{\longrightarrow} \mathrm{H}_{2} \mathrm{O}_{2}
$$

在人体组织中, 内源性的 $\mathrm{H}_{2} \mathrm{O}_{2}$ 主要来自于线粒体、内质网、唾液细胞、微生物和肺, 外源性有 害刺激如紫外线、外源生物、传染源等能诱发 $\mathrm{H}_{2} \mathrm{O}_{2}$ 产生 ${ }^{[23]}$ 。 $\mathrm{H}_{2} \mathrm{O}_{2}$ 是活性氧(ROS)的一种, 线粒体中 至少有 10 个能够产生 ROS 的位点, 线粒体内的多蛋白质复合体 NADH-泛醌氧化还原酶在 NADH 作用下, 能够产生超氧阴离子. $\mathrm{O}_{2}^{-}$; 在琥珀酸或者三磷酸甘油驱动的反向电子传递中, $\mathrm{NAD}^{+}$被还原 $^{-}$ 成 $\mathrm{NADH}$, 随即产生 $\mathrm{H}_{2} \mathrm{O}_{2}$ 。在内质网中, 蛋白质氧化折叠形成二硫键, 也会生成 ROS。哺乳动物过 氧化物酶体系中, 在氧化酶(如脂酰辅酶 $\mathrm{A}$ 氧化酶、黄嘌呤氧化酶等)的催化下, 某些底物(如脂肪酸、 黄嘌呤、尿酸等)和分子氧发生质子转移, 底物被氧化代谢同时有 $\mathrm{H}_{2} \mathrm{O}_{2}$ 和 $\cdot \mathrm{O}_{2}^{-}$产生。在肝脏和肠道中, 黄嘌呤氧化还原酶 (XOR) 可催化还原 $\mathrm{O}_{2}$ 产生 $\cdot \mathrm{O}_{2}^{-}$和 $\mathrm{H}_{2} \mathrm{O}_{2}$ 。所有的血管细胞都产生 ROS, 其中 NADPH 氧化酶发挥主要作用。

在植物细胞内, $\mathrm{H}_{2} \mathrm{O}_{2}$ 大部分来源于线粒体、叶绿体、细胞膜和过氧化物酶体, $\mathrm{H}_{2} \mathrm{O}_{2}$ 通过电子途 经和代谢产生, 叶绿体和线粒体是主要的产生部位。能量过剩或者叶绿体和线粒体的能量代谢紊乱 是植物细胞产生 $\cdot \mathrm{O}_{2}^{-}$的关键原因, $\cdot \mathrm{O}_{2}^{-}$经过酶还原生成 $\mathrm{H}_{2} \mathrm{O}_{2}$ 。叶绿体中, $\mathrm{H}_{2} \mathrm{O}_{2}$ 来自于光合电子传递系 统, 在叶绿体光合电子传递链 PSI 的受体端有大量的自动氧化酶类存在, 能够通过米勒反应将 $\mathrm{O}_{2}$ 还原成超氧阴离子. $\mathrm{O}_{2}^{-}$, 通过不同的超氧化物歧化酶(SODs)还原或自发分解, $\cdot \mathrm{O}_{2}^{-}$迅速转化为 $\mathrm{H}_{2} \mathrm{O}_{2}$ 。 线粒体电子传递链中, 电子本应被传递至末端氧化酶, 再与 $\mathrm{O}_{2}$ 和质子反应形成 $\mathrm{H}_{2} \mathrm{O}$, 但是非血红素 铁蛋白、黄素蛋白、醌、半醌类分子, 它们处于呼吸链底物端, 可使这一过程发生障碍, 部分电子未传 递到末端氧化酶而成为渗漏电子, 被 $\mathrm{O}_{2}$ 捕获, 直接反应生成 $\cdot \mathrm{O}_{2}^{-}$, 再经酶促反应, 生成 $\mathrm{H}_{2} \mathrm{O}_{2}{ }^{[24,25]}$ 。 据估计, 进入植物呼吸链的 $\mathrm{O}_{2}$ 有约 $1 \%-5 \%$ 被转化为 $\mathrm{H}_{2} \mathrm{O}_{2}$ 。 


\section{$5.2 \mathrm{H}_{2} \mathrm{O}_{2}$ 的生物学功能}

与其他 $\mathrm{ROS}$ 相比, $\mathrm{H}_{2} \mathrm{O}_{2}$ 较稳定、寿命较长, 在机体内半衰期约为 $1 \mathrm{~ms}$, 易在不同的细胞腔室 间跨膜扩散。在 $\mathrm{nmol} \cdot \mathrm{L}^{-1}$ 低浓度级别的 $\mathrm{H}_{2} \mathrm{O}_{2}$ 可作为信号分子参与调节生命体细胞的增殖、分化、能 量代谢、基因表达、传导缺氧信号、介导免疫反应, 以及促调亡或抗调亡等许多生理过程。高浓度 的 $\mathrm{H}_{2} \mathrm{O}_{2}$ 会攻击细胞核酸、蛋白质、脂质等大分子, 干扰 ATP 和激素合成、细胞生长等, 造成细胞 损伤, 触发氧化伤害; 可干扰信号传递, 能诱导细胞程序性死亡和细胞周期停滞, 也可参与受损、 突变、调亡细胞的清除, 能促进吞噬细胞发挥吞噬作用, 杀死侵入体内的病毒、细菌、真菌等微生

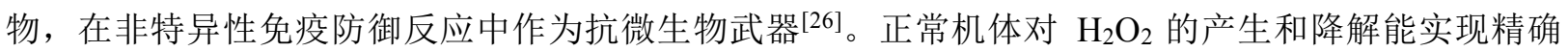
调控。在微生物、紫外线、害虫、高盐等极端条件刺激下, 会引起 $\mathrm{H}_{2} \mathrm{O}_{2}$ 和其他 ROS 的大量积累, 细 胞产生氧化胁迫响应, 启动体内其他信号级联。在生物防御下, 过量的 $\mathrm{H}_{2} \mathrm{O}_{2}$ 会被细胞内的过氧化氢 酶、谷胱甘肽过氧化物酶(GSH-Px)、过氧化物酶和多酚氧化酶等清除 ${ }^{[27]}$ 。

人类常见疾病，如肿瘤、心脏病、骨关节炎、风湿性关节炎、糖尿病以及神经退化性问题如阿 尔兹海默症、帕金森病, 甚至衰老的自身过程, 其产生和加速发展的部分原因都与 ROS 水平异常升 高有关 ${ }^{[28]}$ 。例如, 肿瘤细胞代谢光奋, 氧化还原平衡受损, 高浓度 $\mathrm{H}_{2} \mathrm{O}_{2}$ 是肿瘤微环境的标志之一 ${ }^{[29]}$; 此外, $\mathrm{H}_{2} \mathrm{O}_{2}$ 通过对钙离子和蛋白质磷酸信号的传导可维持血管收缩和舒张的自稳态, 但在动脉弹样 硬化的症状里, 高浓度 $\mathrm{H}_{2} \mathrm{O}_{2}$ 剧烈的氧化刺激会引发血管损伤和细胞死亡。 $\mathrm{H}_{2} \mathrm{O}_{2}$ 及氧化应激状态已 作为多种疾病的诊断指标, 并发展出预防和治疗疾病的新靶点、新手段 ${ }^{[30-32]}$ 。

\section{$6 \mathrm{H}_{2} \mathrm{O}_{2}$ 的检测方法}

对环境、食品以及生命体中 $\mathrm{H}_{2} \mathrm{O}_{2}$ 的精确定量检测具有重要的实践意义。检测 $\mathrm{H}_{2} \mathrm{O}_{2}$ 的方法有多 种, 如滴定法、光度法、生物成像技术、电化学法等。滴定法利用传统的定量分析原理, 此不赘述。 主要介绍后三种检测方法。

\section{1 紫外分光光度法}

利用催化剂使 $\mathrm{H}_{2} \mathrm{O}_{2}$ 均裂为强氧化性自由基, 进而将 $N, N$-二乙基对苯二胺(DPD)氧化生成紫红色 的阳离子自由基· $\mathrm{DPD}^{+}$(图 3), $\cdot \mathrm{DPD}^{+}$在波长 510 和 $551 \mathrm{~nm}$ 有强吸收, 用紫外可见分光光度法测定并 推出 $\mathrm{H}_{2} \mathrm{O}_{2}$ 含量。亦可用纳米 $\mathrm{Fe}_{3} \mathrm{O}_{4}$ 代替辣根过氧化物酶( $\mathrm{HRP}$ )做催化剂, 测定雨水中 $\mathrm{H}_{2} \mathrm{O}_{2}$ 含量, 结 果与 HRP-DPD 经典方法一致 ${ }^{[33]}$ 。

\section{$6.2 \mathrm{H}_{2} \mathrm{O}_{2}$ 检测与生物成像技术}

活体细胞内 $\mathrm{H}_{2} \mathrm{O}_{2}$ 的细微变化可通过生物成像技术实现动态检测, 可使用化学选择性苂光探针、 荧光蛋白等, 选择性、针对性地对生物体内的 $\mathrm{H}_{2} \mathrm{O}_{2}$ 进行定位定量。所用生物成像技术包括化学/生 物发光成像技术、激光共聚焦显微镜检测技术、双光子显微镜检测技术等 ${ }^{[34]}$ 。

苯硼酸酯与 $\mathrm{H}_{2} \mathrm{O}_{2}$ 可发生特异性化学选择性反应而生成苯酚, 已被广泛用作检测 $\mathrm{H}_{2} \mathrm{O}_{2}$ 的苂光探 针。Tang 等 $\left.{ }^{[35}\right]$ 发展了聚集诱导荧光探针四苯基乙烯嗍酸酯类化合物(TPE-BO), 在 $\mathrm{H}_{2} \mathrm{O}_{2}$ 存在下, 苯 碀酸酯被转化为苯酚基团(图 4), 因而聚集导致在 $500 \mathrm{~nm}$ 呈强荧光。在 $10-200 \mu \mathrm{mol} \cdot \mathrm{L}^{-1}$ 范围内,

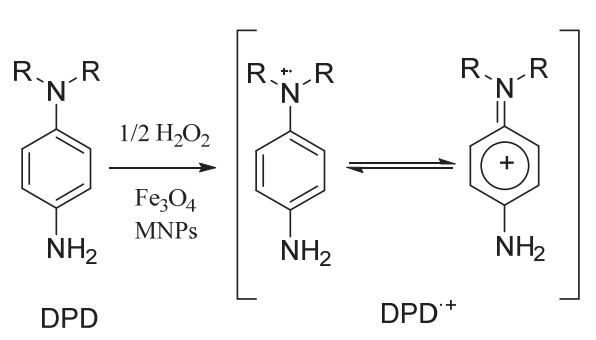

图 $3 \mathrm{DPD}$ 与 $\mathrm{H}_{2} \mathrm{O}_{2}$ 的反应

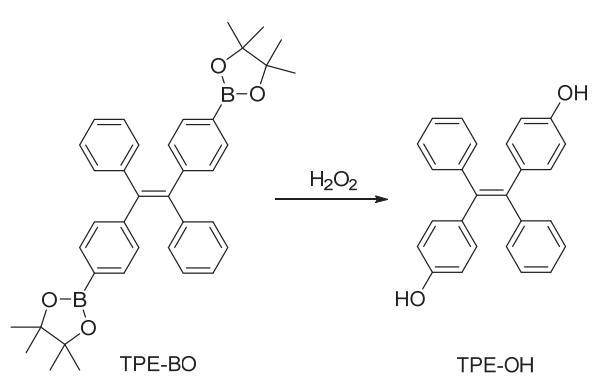

图 4 TPE-BO 与 $\mathrm{H}_{2} \mathrm{O}_{2}$ 的反应 
$\mathrm{H}_{2} \mathrm{O}_{2}$ 浓度和探针荧光强度呈线性相关, $\mathrm{H}_{2} \mathrm{O}_{2}$ 的检出限可达 $0.52 \mu \mathrm{mol} \cdot \mathrm{L}^{-1}$ 。此法对 $\mathrm{H}_{2} \mathrm{O}_{2}$ 具有高选择 性, 已应用于巨噬细胞中 $\mathrm{H}_{2} \mathrm{O}_{2}$ 的监测, 而对其他 $\mathrm{ROS} / \mathrm{RNS}$ 物种, 包括· $\mathrm{OH} 、 \mathrm{HOCl} 、 \mathrm{NO} 、{ }^{1} \mathrm{O}_{2} 、 \mathrm{O}_{2}^{-}$ 和 $\mathrm{ONOO}^{-}$诱导的荧光增强很弱, 可忽略不计。

\section{3 电化学法}

由纳米 $\mathrm{Fe}_{3} \mathrm{O}_{4}$ 粒子、氧化石墨烯 $(\mathrm{GO})$ 和石墨烯(PG)制备的复合材料 $\mathrm{Fe}_{3} \mathrm{O}_{4} / \mathrm{GO} / \mathrm{PG} 0.88$ 传感器对 $\mathrm{H}_{2} \mathrm{O}_{2}$ 浓度的变化非常敏感, 是检测 $\mathrm{H}_{2} \mathrm{O}_{2}$ 的理想电极材料。它与 $\mathrm{H}_{2} \mathrm{O}_{2}$ 浓度有两个线性范围: 一个为 17.00 至 $277.00 \mu \mathrm{mol} \cdot \mathrm{L}^{-1}$, 灵敏度为 $0.31 \mu \mathrm{A} \cdot \mu \mathrm{mol}^{-1} \cdot \mathrm{L}$; 另一个为 0.50 至 $17.00 \mu \mathrm{mol} \cdot \mathrm{L}^{-1}$, 灵敏度为 $0.18 \mu \mathrm{A} \cdot \mu \mathrm{mol}^{-1} \cdot \mathrm{L}$, 检测限为 $0.09 \mu \mathrm{mol} \cdot \mathrm{L}^{-1}$ 。生物体系中 $\mathrm{Cl}^{-} 、 \mathrm{Na}^{+} 、 \mathrm{Fe}^{3+}$ 或 $\mathrm{K}^{+}$会影响传感器的响应, 但不会影响 $\mathrm{H}_{2} \mathrm{O}_{2}$ 的测定, 故选择性较好 ${ }^{[36]}$ 。

此外, 由玻碳电极、壳聚糖-多壁碳纳米管、金纳米粒子以及血红蛋白制备的 $\{\mathrm{Hb} / \mathrm{GNPs}\}_{n} / \mathrm{CS}-$ $\mathrm{MWNTs} / \mathrm{GC}$ 复合电极 ${ }^{[37]}$, 由十二烷基硫酸钠(SDS)-多壁碳纳米管(MWCNTs)修饰的玻碳电极 ${ }^{[38]}$, 由 还原氧化石墨烯/银纳米颗粒(rGO/AgNPs)修饰的玻碳电极 [39]等, 也对 $\mathrm{H}_{2} \mathrm{O}_{2}$ 具有良好的电化学响应, 均可用于 $\mathrm{H}_{2} \mathrm{O}_{2}$ 电化学生物传感器的开发研究。

\section{$7 \mathrm{H}_{2} \mathrm{O}_{2}$ 的应用}

$\mathrm{H}_{2} \mathrm{O}_{2}$ 分解产物无毒无害, 因而应用广泛。全球每年的 $\mathrm{H}_{2} \mathrm{O}_{2}$ 需求量超过 500 万吨, 其中近 $80 \%$ 应用于洗涤、漂白及纸浆和造纸业。

$\mathrm{H}_{2} \mathrm{O}_{2}$ 是纺织、造纸、皮革、木材、洗涤用品中的漂白剂和去味剂。 $\mathrm{H}_{2} \mathrm{O}_{2}$ 漂白速度快、效果好且 白度稳定, 纤维损害小。在光照、碱性、电离辐射等条件下, $\mathrm{H}_{2} \mathrm{O}_{2}$ 分解产生的高活性的羟基自由基 $(\cdot \mathrm{OH})$, 在纸浆漂白中, $\cdot \mathrm{OH}$ 攻击木素的醛基发色基团使其功能失效, 并阻止有色物的再生, 实现永 久褪色。碱性介质中 $\mathrm{H}_{2} \mathrm{O}_{2}$ 电离生成的过氧氢阴离子 $\left(\mathrm{HO}_{2}^{-}\right)$也是活性漂白因子, 主要破坏木素中的羰 基结构。

化学工业中, $\mathrm{H}_{2} \mathrm{O}_{2}$ 是生产多种化学试剂如过氧化钙、过氧乙酸、邻苯二酚、甘油、二甲基亚砜、 环氧葵花籽油等的原料。在有机合成领域, $\mathrm{H}_{2} \mathrm{O}_{2}$ 是最重要的 $\mathrm{C}-\mathrm{H}$ 和 $\mathrm{C}=\mathrm{C}$ 等官能团的氧化试剂。

在食品工业中, $\mathrm{H}_{2} \mathrm{O}_{2}$ 用于食物、容器和包装材料的消毒, 也用于面包发酵、食物漂白等。各种 日用品如化妆品、染发剂、家用清洁剂等也含有 $\mathrm{H}_{2} \mathrm{O}_{2}$ 。

在医疗中, $3 \% \mathrm{H}_{2} \mathrm{O}_{2}$ 稀溶液是医用消毒剂, 用于伤口消毒。它不仅破坏细菌和病毒, 也会侵蚀 局部细胞使组织坏死, 使创面处血管蛋白质和血小板凝滞, 诱发血管收缩, 达到良好的止血消毒效 果。 $\mathrm{H}_{2} \mathrm{O}_{2}$ 处理深伤口效果更佳, 创口细胞内的生物酶催化 $\mathrm{H}_{2} \mathrm{O}_{2}$ 迅速分解产生大量氧气, 能有效清 除伤口里的坏死组织、灰尘、脓等污物, 防止更严重的感染, 同时生成的氧气能抑制伤口深处的厌

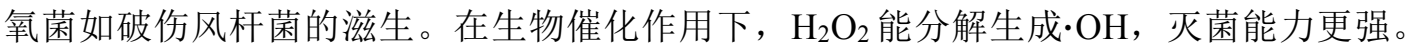

环境治理中, $\mathrm{H}_{2} \mathrm{O}_{2}$ 能够消解 $\mathrm{NO}$ 、酚类、醛类、重金属、硫氰酸盐、硝酸盐和次氯酸盐等有毒 化学物质和细菌、真菌、芽孢等微生物, 用于处理工业和生活废水、气体净化和土壤治理。 $\mathrm{H}_{2} \mathrm{O}_{2}$ 能 破坏微生物细胞屏障结构, 增加细胞膜通透性; 对细菌体内半胱氨酸颈基进行可逆氧化而使蛋白质 和酶的结构及功能受到损伤; 对菌体磷酸二酯键和脱氧核糖的作用使 DNA 核苷酸链发生断裂和碱 基组成发生改变……多重积累的氧化损伤, 致使微生物细胞环境发生溃乱直至死亡, 达到杀菌目的。 $\mathrm{H}_{2} \mathrm{O}_{2}$ 可用于处理湖泊、水池里的蓝藻。 $\mathrm{H}_{2} \mathrm{O}_{2}$ 能阻止蓝藻的光合过程, 引起蓝藻种群崩溃, 破坏蓝藻 产生的大部分微囊藻毒素(MCs), 破坏色素合成和膜结构的完整性, 导致细胞死亡 ${ }^{[40]}$ 。通过电化学方 法原位产 $\mathrm{H}_{2} \mathrm{O}_{2}$ 用于处理环境有害污染物, 具有投资少、效率高、在环境治理中显示了极大的潜力。

在电子工业中, $\mathrm{H}_{2} \mathrm{O}_{2}$ 作为电子仪表元件的清洗剂、防腐液并用于电镀液的处理等, 使用浓度一 般为 70\%-90\%。在冶金工业中, $\mathrm{H}_{2} \mathrm{O}_{2}$ 可用于钴、铀等金属的提炼。在军工领域, $\mathrm{H}_{2} \mathrm{O}_{2}$ 用作鱼雷、 火箭、潜艇、飞机的氧化剂和推进剂; $\mathrm{H}_{2} \mathrm{O}_{2}$ 作为燃料助燃剂, 高温、高浓度(90\%-98\%)条件下用重 金属催化, 剧烈产生用于推进的热量和氧气, 冲击力强劲而迅猛。 


\section{8 使用安全}

$\mathrm{H}_{2} \mathrm{O}_{2}$ 不稳定, 浓度越大越容易分解。光照、温度、 $\mathrm{pH}$ 、过渡金属离子、生物酶、溶液浓度等均 能影响 $\mathrm{H}_{2} \mathrm{O}_{2}$ 的分解。太阳光线 $\left(320-380 \mathrm{~nm}\right.$ ) 会加速 $\mathrm{H}_{2} \mathrm{O}_{2}$ 分解。温度越高, $\mathrm{H}_{2} \mathrm{O}_{2}$ 分解越快, 在 $30^{\circ} \mathrm{C}$ 时一年只分解 $1 \%$, 而在 $100{ }^{\circ} \mathrm{C}$ 时一天就能分解 $2 \%$, 在 $140{ }^{\circ} \mathrm{C}$ 时 $\mathrm{H}_{2} \mathrm{O}_{2}$ 即沸腾分解放出气体。 $\mathrm{H}_{2} \mathrm{O}_{2}$ 在 $\mathrm{pH}$ 为 3.5-4.5 时最稳定, 强酸 $(\mathrm{pH}<3)$ 或 $\mathrm{pH}=5-7$ 条件下, 分解有所加速。在碱性条件 $(\mathrm{pH}>11)$ 下, $\mathrm{H}_{2} \mathrm{O}_{2}$ 溶液中大量形成亲核的过氧氢阴离子 $\mathrm{HO}_{2}^{-}$, 触发 $\mathrm{H}_{2} \mathrm{O}_{2}$ 更迅猛的分解。过渡金属离子可降 低 $\mathrm{H}_{2} \mathrm{O}_{2}$ 的分解活化能, 催化其分解, 如 $\mathrm{Cu}^{2+} 、 \mathrm{Fe}^{2+} 、 \mathrm{Mn}^{2+} 、 \mathrm{Ni}^{2+} 、 \mathrm{Zn}^{2+} 、 \mathrm{Cr}^{3+}$ 等, 其中催化作用最强 的是 $\mathrm{Cu}^{2+}$ 和 $\mathrm{Fe}^{2+}$ 。碱性介质中常有微量重金属离子, 所以 $\mathrm{H}_{2} \mathrm{O}_{2}$ 在碱性介质中分解更快。过氧化物 酶催化 $\mathrm{H}_{2} \mathrm{O}_{2}$ 的分解主要发生在生物体内, 催化效率是 $\mathrm{Fe}^{2+}$ 的 $10^{7}$ 倍, 能极为快速地将生物代谢产生 的 $\mathrm{H}_{2} \mathrm{O}_{2}$ 消耗分解, 高效的酶促反应在生物应对环境胁迫和维持生物细微结构的氧化还原稳态中扮 演极为关键的角色。

因此, 为防止 $\mathrm{H}_{2} \mathrm{O}_{2}$ 的分解, 降低损耗, 要使用避光的塑料容器存储在阴凉处, 并留有排气孔使 分解生成的气体能及时排出, 储存浓度不宜过高。常加入稳定剂, 如锡酸钠、焦硫酸钠、8-羟基喹 啉、乙酸、过氧乙酸、磷酸等。

$\mathrm{H}_{2} \mathrm{O}_{2}$ 本身不燃, 但与可燃物反应放出大量热量和氧气而引起着火爆炸。它可与许多有机物如糖、 淀粉、醇类、石油产品等形成爆炸性混合物, 在撞击、受热或电火花作用下能发生爆炸。 $\mathrm{H}_{2} \mathrm{O}_{2}$ 与许 多无机化合物或杂质接触后会迅速分解而导致爆炸, 放出大量的热量、氧和水蒸气。大多数重金属 及其氧化物和盐类都是活性催化剂, 尘土、香烟灰、碳粉、铁锈等也能加速其分解。浓度超过 $74 \%$ 的 $\mathrm{H}_{2} \mathrm{O}_{2}$, 在具有适当的点火源或温度的密闭容器中, 能产生气相爆炸。

高浓度的 $\mathrm{H}_{2} \mathrm{O}_{2}$ 腐蚀性强, 危险性大, 只在工业上使用。30\%的 $\mathrm{H}_{2} \mathrm{O}_{2}$ 接触皮肤或眼睛可引起灼 伤甚至失明, 吞服后会严重导致肠胃刺激和溃疡, 对人体伤害巨大。 $\mathrm{H}_{2} \mathrm{O}_{2}$ 蒸汽进入呼吸道, 也会引 起呼吸道刺激, 国家标准规定空气中的 $\mathrm{H}_{2} \mathrm{O}_{2}$ 含量不得高于 $1.4 \mathrm{mg} \cdot \mathrm{m}^{-3}$ 。即使是食品级的 $\mathrm{H}_{2} \mathrm{O}_{2}$, 也 只是消毒剂, 消毒之后残留 $\mathrm{H}_{2} \mathrm{O}_{2}$ 还需要添加过氧化氢酶催化分解之, 还需通过痕量检测确认其完全 清除后方能出库, 确保食品安全。医用级低浓度 $\mathrm{H}_{2} \mathrm{O}_{2}$ 倘若进入肠胃, 分解产生的活性自由基在胃肠 内积聚会引起化学性胃炎或结肠炎 ${ }^{[41]}$, 故用 $3 \% \mathrm{H}_{2} \mathrm{O}_{2}$ 漱口时一定要稀释 3 倍, 并防止吞咽。医用 $\mathrm{H}_{2} \mathrm{O}_{2}$ 消毒剂不能用于正常皮肤。 $\mathrm{H}_{2} \mathrm{O}_{2}$ 接触正常皮肤会发生氧化反应使皮肤发白, 造成局部损伤, 加 速细胞老化。

\section{9 结语}

$\mathrm{H}_{2} \mathrm{O}_{2}$ 结构微妙, 用途广泛, 它已悄无声息地渗入各行各业, 在漂白、医药、化工、环保、日用 品、食品工业、火箭燃料等行业发挥至关重要的作用。随着科学研究的深入, $\mathrm{H}_{2} \mathrm{O}_{2}$ 的理化性质已经 清楚, 未来研究将聚焦于如何实现 $\mathrm{H}_{2} \mathrm{O}_{2}$ 的高效清洁生产、超高纯 $\mathrm{H}_{2} \mathrm{O}_{2}$ 的制备以及原位制备与应用; 如何有效检测食品、水和空气等环境中的残留 $\mathrm{H}_{2} \mathrm{O}_{2}$, 以及如何探测生物活体内 $\mathrm{H}_{2} \mathrm{O}_{2}$ 聚积位点和浓 度大小; 探索其在生命体复杂的调控网络中, 如何实现精准调控细胞的生长、发育和应激反应等复 杂生理过程; 以及 $\mathrm{H}_{2} \mathrm{O}_{2}$ 在肿瘤等重大疾病中作为诊断指标和治疗靶点的研究与应用。

\section{参 考 文 献}

[1] De Grey, A. D. N. J. DNA Cell Biol. 2002, 21 (4), 251.

[2] Richard, L. M. The 100 Most Important Chemical Compounds: a Reference Guide, 1st ed.; Greenwood Press: Connecticut, USA, 2007; pp. $144-146$.

[3] Koppenol, W. H.; Sies, H. Nature 2018, 559 (7713), 181.

[4] Liang, M. C.; Hartman, H.; Kopp, R. E.; Kirschvink, J. L.; Yung, Y. L. P. Natl. Acad. Sci. USA 2006, 103 (50), 18896.

[5] Mostofa, K. M. G.; Yoshioka, T.; Mottaleb, A.; Vione, D. Photobiogeochemistry of Organic Matter, Principles and Practices in Water 
Environments, 1st ed.; Springer: Heidelberg, Germany, 2013; pp. 139-207.

[6] Pesterfield, L. J. Chem. Educ. 2009, 86 (10), 1182

[7] Busing, W. R.; Levy, H. A. J. Chem. Phys. 1965, 42 (9), 3054.

[8] Giguere, P. A. J. Chem. Educ. 1983, 60 (5), 399.

[9] Talsi, E. P.; Bryliakov, K. P. Coordin. Chem. Rev. 2012, 256 (13-14), 1418.

[10] Burek, B. O.; Bormann, S.; Hollmann, F.; Bloh, J. Z.; Holtmann, D. Green Chem. 2019, 21 (12), 3232.

[11] Ranganathan, S.; Sieber, V. Catalysts 2018, 8 (9), 379.

[12] Campos-Martin, J. M.; Blanco-Brieva, G.; Fierro, J. L. G. Angew. Chem. Int. Edit. 2006, 45 (42), 6962.

[13] 胡长诚. 化学推进剂与高分子材料, 2003, 19 (1), 14.

[14] Tomita, O.; Otsubo, T.; Higashi, M.; Ohtani, B.; Abe, R. ACS Catal. 2016, 6 (2), 1134.

[15] Kimura, M.; Yamamoto, M.; Nagai, A. Inorg. Chim. Acta 1986, 117 (2), 169.

[16] Perry, S. C.; Pangotra, D.; Vieira, L.; Csepei, L. I.; Sieber, V.; Wang, L.; Ponce de Leon, C.; Walsh, F. C. Nat. Rev. Chem. 2019, 3 (7), 442.

[17] Shiraishi, Y.; Kanazawa, S.; Kofuji, Y.; Sakamoto, H.; Ichikawa, S.; Tanaka, S.; Hirai, T. Angew. Chem. Int. Edit. 2014,53 (49), 13454.

[18] Liu, J.; Zou, Y.; Jin, B.; Zhang, K.; Park, J. H. ACS Energy Lett. 2019, 4 (12), 3018.

[19] Ranganathan, S.; Sieber, V. Catalysts 2018, 8 (9), 379.

[20] Freakley, S. J.; He, Q.; Harrhy, J. H.; Lu, L.; Crole, D. A.; Morgan, D. J.; Ntainjua, E. N.; Edwards, J. K.; Carley, A. F.; Borisevich, A. Y.; et al. Science 2016, 351 (6276), 965

[21] Xia, C.; Xia, Y.; Zhu, P; Fan, L.; Wang, H. T. Science 2019, 366 (6462), 226.

[22] Zhang, M. F.; Hao, J. C.; Neyman, A.; Wang, Y. F.; Weinstock, I. A. Inorg. Chem. 2017, 56 (5), 2400.

[23] Kim, H. J. J. Microbiol. Biotechn. 2014, 24 (11), 1455.

[24] Cerny, M.; Habanova, H.; Berka, M.; Luklova, M.; Brzobohaty, B. Int. J. Mol. Sci. 2018, 19 (9), 2812.

[25] Lennicke, C.; Rahn, J.; Lichtenfels, R.; Wessjohann, L. A.; Seliger, B. Cell Commun. Signal. 2015, 13 (1), 39.

[26] Filomeni, G.; De Zio, D.; Cecconi, F. Cell Death Differ. 2015, 22 (3), 377.

[27] Wang, Y.; Branicky, R.; Noe, A.; Hekimi, S. J. Cell Biol. 2018, 217 (6), 1915

[28] Chaiswing, L.; St. Clair, W. H.; St. Clair, D. K. Antioxid. Redox Sign. 2018, 29 (13), 1237.

[29] Weinberg, F.; Ramnath, N.; Nagrath, D. Cancer-Am. Cancer Soc. 2019, 11 (8), 1191.

[30] Glasauer, A.; Chandel, N. S. Biochem. Pharmacol. 2014, 92 (1), 90

[31] Rajendran, P.; Nandakumar, N.; Rengarajan, T.; Palaniswami, R.; Gnanadhas, E. N.; Lakshminarasaiah, U.; Gopas, J.; Nishigaki, I. Clin. Chim. Acta 2014, 436,332

[32] Fransen, M.; Lismont, C. Antioxid. Redox Sign. 2019, 30 (1), 95.

[33] 常青，江国栋，唐和清，崔龙哲，吕康乐，吴来燕. 实验室科学, 2014, 17 (2), 27.

[34] Chen, X. Q.; Wang, F.; Hyun, J. Y.; Wei, T. W.; Qiang, J.; Ren, X. T.; Shin, I.; Yoon, J. Chem. Soc. Rev. 2016, 45 (10), 2976.

[35] Zhang, W.; Liu, W.; Li, P.; Huang, F.; Wang, H.; Tang, B. Anal. Chem. 2015, 87 (19), 9825.

[36] Cai, L. L.; Hou, B. J.; Shang, Y. Y.; Xu, L.; Zhou, B.; Jiang, X. N.; Jiang, X. Q. Chem. Phys. Lett. 2019, 736, 136797.

[37] Chen, S. H.; Yuan, R.; Chai, Y. Q.; Yin, B.; Xu, Y. Electroanal. 2008, 20 (19), 2141.

[38] Zhang, J.; Gao, L. Mater. Lett. 2007, 61 (17), 3571.

[39] 刘杰, 赵振廷, 史健芳, 张文栋, 胡杰. 材料导报, 2016, $30(24), 11$.

[40] Spoof, L.; Jaakkola, S.; Vazic, T.; Häggqvist, K.; Kirkkala, T.; Ventelä, A. M.; Kirkkala, T.; Svircev, Z.; Meriluoto, J. Environ. Sci. Pollut. R. 2020, $27(8), 8638$

[41] Zanelli, M.; Ragazzi, M.; De Marco, L. Br. J. Clin. Pharmacol. 2017, 83 (2), 427. 